Check for updates

Cite this: RSC Adv., 2018, 8, 6620

Received 16th October 2017

Accepted 23rd January 2018

DOI: $10.1039 / c 7 r a 11420 f$

rsc.li/rsc-advances

\section{Comparative pharmacokinetics of four active components on normal and diabetic rats after oral administration of Gandi capsules $\uparrow$}

\author{
Renjie Xu, (D) $\ddagger^{a}$ Jia Qi, $\stackrel{t}{a}^{a}$ Ruan-Juan Zhan, ${ }^{b}$ Gui-Sheng Zhou, ${ }^{c}$ Bin Hao, ${ }^{d}$ Jing Ma, ${ }^{a}$ \\ Xin Wei, ${ }^{a}$ A.-Jing $\mathrm{Xu}^{\star a}$ and Jian Zhang ${ }^{\star a}$
}

The Gandi capsule, a famous traditional Chinese medicine (TCM), is a hospital preparation that has been widely used in China for decades for the treatment of diabetes. The aim of this study is to compare the pharmacokinetics of four of the active components of Gandi capsules, which are the primary antidiabetic ingredients, on normal and diabetic Sprague-Dawley rats following oral administration of the capsules. Baicalin, wogonoside, wogonin, loganin and puerarin (internal standard) were prepared using methanol precipitation, and the separation of the five components was achieved through a ZORBAX Eclipse Plus $\mathrm{C}_{18}$ column by gradient elution using water (containing $0.1 \%$ formic acid) and acetonitrile as the mobile phase. After oral administration of Gandi capsules to the normal and diabetic rats, plasma was harvested and analyzed using liquid chromatography coupled with tandem mass spectrometry, and the primary pharmacokinetic parameters were calculated by DAS 2.0. Compared with the normal group, some pharmacokinetic parameters especially the $\mathrm{AUC}_{0-48 \mathrm{~h}}$ of the four compounds significantly increased in the diabetic groups. The results demonstrated that the four constituents in normal and diabetic rats had obvious differences in some pharmacokinetic characteristics, suggesting that the rate and extent of drug metabolism were altered in diabetic animals. The results could be helpful for demonstrating the compatibility mechanism and providing clinical medication guidance for Gandi capsules.

\section{Introduction}

Traditional Chinese Medicines (TCMs) are mostly prescribed in combinations in accordance with the theory of obtaining synergistic effects or reducing possible adverse reactions. The Gandi capsule, a famous traditional Chinese medicine, that consists of radix rehmanniae, scutellaria, astragalus, cornus officinalis, leonurus, muscardine silkworm, sophora flower and phyllanthus (Shengdi, Huangqin, Huangqi, Shanzhuyu, Yimucao, Jiangcan, Huaihua and Yuganzi in Chinese, respectively), has been used to treat diabetes for decades. Wei et al.

\footnotetext{
${ }^{a}$ Department of Pharmacy, Xinhua Hospital, No. 1665 Kongiiang Road, Yangpu District, Shanghai 200092, China. E-mail: zhangjian@xinhuamed.com.cn; ajingxu@ xinhuamed.com.cn; Fax: +86-21-25077150; Tel: +86-21-25077150, +86-21-25077156 ${ }^{b}$ Department of Pharmacy, The First Affiliated Hospital, Wenzhou Medical University, Wenzhou, China

'Jiangsu Collaborative Innovation Center of Chinese Medicinal Resources Industrialization, National and Local Collaborative Engineering Center of Chinese Medicinal Resources Industrialization and Formulae Innovative Medicine, Nanjing University of Chinese Medicine, No. 138 Xianlin Road, Nanjing 210023, China

${ }^{d}$ School of Pharmacy, Shanghai Jiao Tong University, No. 800 Dongchuan Road, Minhang District, Shanghai 200240, China

$\dagger$ Electronic supplementary information (ESI) available. See DOI: 10.1039/c7ra11420f

‡ Contributed equally.
}

reported that after 4 months of treatment of Gandi capsules for Diabetic Nephropathy, clinical efficacy of trial group subjects was better than that of the control group, with statistical significance $(P<0.05){ }^{1}$ Zhu et al. found Gandi capsules can control obstinate hyper-glycemia, and improve fat metabolism as well as hemorheological parameters. ${ }^{2}$

Flavonoid glycosides and cyclopropene, which contains baicalin, wogonoside, wogonin and loganin (Fig. 1A-D), were reported to be the main bioactive constituents in Gandi capsule, ${ }^{3}$ and have anti-inflammatory, ${ }^{4,5}$ anti-tumor, ${ }^{6-9}$ antioxidant activities ${ }^{10-12}$ and especially have the potential for the treatment of diabetes and diabetic complications. ${ }^{13-17}$

A comprehensive characterization of pharmacokinetic characteristic of TCM is vital not only to gaining insight into its in vivo action and mechanism but also to carrying out further clinical studies. The analysis of mono ingredients or single herbs may not represent the in vivo process of the whole composite prescription. Thus, it is better to investigate the herbal compatibility mechanism of multiple components or the whole prescription rather than single herbs or single compound..$^{18,19}$ Some articles have reported about the in vivo process of baicalin, wogonoside, wogonin, and loganin respectively or in other compound preparations. ${ }^{20-23}$ However, few analytical methods were developed for simultaneous determination of the four 


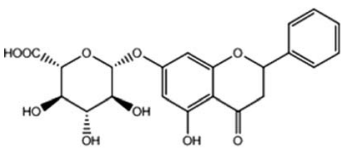

(A)

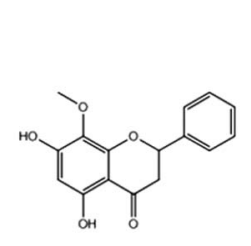

(C)

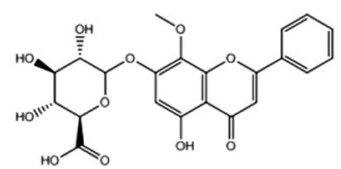

(B)

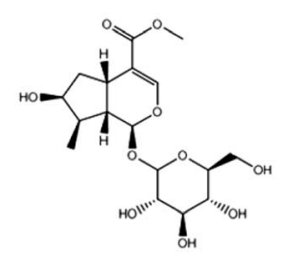

(D)

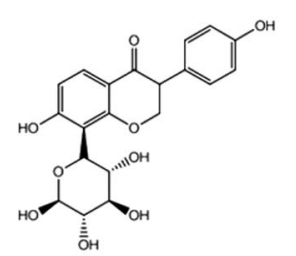

(E)
Fig. 1 Structures of baicalin (A), wogonoside (B), wogonin (C), loganin (D) and puerarin (E; IS).

compounds in rat plasma and to describe the profile of Gandi capsule in vivo after oral administration.

Most of the existing pharmacokinetic data of traditional Chinese medicine came from studies with healthy animals or healthy human subjects. Unfortunately, under pathological conditions such as diabetes, enzyme activity and membrane transporter capacity are often altered from the normal conditions, ${ }^{24,25}$ leading to changes in the pharmacokinetic and metabolic behaviors of drugs. For example, Zeng et al. reported that compared to normal rats, maximum concentration $\left(C_{\max }\right)$ and area under the curve $\left(\mathrm{AUC}_{0-10 \mathrm{~h}}\right)$ values of oral arctigenin in diabetic rats increased by $356.8 \%$ and $223.4 \%$, respectively. ${ }^{26}$ Another report showed that the absorptions of six flavonoids in Maydis stigma in diabetic rats were all significantly higher than those in the normal group, which provides an experimental basis for the role of Maydis stigma in anti-diabetic treatment. ${ }^{27}$

In this study, a sensitive, specific, rapid, and precise approach was developed with a short retention time (less than $4.3 \mathrm{~min}$ ) and acceptable low limit of determination (LLOQ) by optimized MS conditions for the simultaneously determination of four constituents in rat plasma and was applied to compare the pharmacokinetics of these constituents after oral administration of Gandi capsule between normal and diabetic rats, and furthermore to investigate the herbal compatibility mechanism.

\section{Materials and methods}

\section{Chemicals, reagents and animals}

Baicalin, wogonoside, wogonin, loganin and puerarin (internal standard, IS) (Fig. 1E) were obtained from the National Institute for the Control of Pharmaceutical and Biological Products (Beijing, China). The purity of these reference standards was greater than $98 \%$. Gandi capsule (40 capsules, $0.3 \mathrm{~g}$ per capsule, batch number: 20161202) were supplied by Fangxin Pharmaceutical Co., Ltd. (Shanghai, China). Acetonitrile and methanol, high-performance liquid chromatography (HPLC) grade, were purchased from Merck Company (Darmstadt, Germany). Ultrapure water for the LC mobile phase was prepared in-house using a Milli-Q system (Millipore, Bedford, MA, USA). All the other reagents were of analytical grade. Streptozotocin (STZ, purity $>98 \%$ ) was purchased from Sigma (Saint Louis, MO, USA).

Male Sprague-Dawley (SD) rats $(250 \pm 20 \mathrm{~g})$ were obtained from Slaccas Experiment Animal Company (Shanghai, China). The rats were maintained in an air-conditioned room under constant room temperature $\left(23{ }^{\circ} \mathrm{C} \pm 2{ }^{\circ} \mathrm{C}\right)$, relative humidity level $(50 \% \pm 15 \%)$, and lighting (12 h light/dark cycle) with free access to food and water for 7 days before the experiment.

\section{Apparatus and LC-MS/MS conditions}

The assay was performed with a Waters UPLC system with an Applied Biosystem 5500 QTRAP ${ }^{\circledR}$ hybrid triple-quadrupole mass spectrometer (Applied Biosystems/MDS Sciex, Foster City, CA, USA) with an electrospray ionization (ESI) interface that was usable in either the positive or the negative-ionization mode. The sample was separated on a ZORBAX Eclipse Plus $\mathrm{C}_{18}$ $(50 \mathrm{~mm} \times 2.1 \mathrm{~mm}, 1.8 \mu \mathrm{m})$.

Samples were eluted through the column with a gradient of water-formic acid (100:0.5, v/v) and acetonitrile ( 0 min, $95: 5$; $3.1 \mathrm{~min}, 37: 63 ; 3.2 \mathrm{~min}, 5: 95 ; 4.3 \mathrm{~min}, 5: 95)$ at a flow rate of $0.4 \mathrm{~mL} \min ^{-1}$ at $45^{\circ} \mathrm{C}$.

The MS parameters for the four active components and IS were optimized in the positive mode. Quantification was performed using multiple-reaction monitoring (MRM) mode. The monitoring parameters, collision energies (CE), and retention times of four standard substances and IS are shown in Table 1. The operating parameters were as follows: ion spray (IS) voltage: $5000 \mathrm{~V}$; temperature: $550{ }^{\circ} \mathrm{C}$; gas 1 and gas 2 (nitrogen) were set at 40 and $40 \mathrm{psi}$, respectively. The curtain gas using nitrogen was set at 40 psi. The dwell time was set at $50 \mathrm{~ms}$ per transition. MS/MS operating conditions were optimized by infusion of the

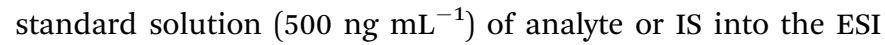
source via a syringe pump, respectively. Data acquisition was

Table 1 List of selected multiple reaction monitoring parameters, collision energies (CE) and retention times of baicalin, wogonoside, wogonin, loganin and puerarin

\begin{tabular}{lllll}
\hline Analytes & $\begin{array}{l}\text { Q1 mass } \\
(\mathrm{Da})\end{array}$ & $\begin{array}{l}\text { Q3 mass } \\
(\mathrm{Da})\end{array}$ & $\begin{array}{l}\text { Collision energies } \\
(\mathrm{eV})\end{array}$ & $\begin{array}{l}\text { Retention times } \\
(\mathrm{min})\end{array}$ \\
\hline Baicalin & 447.0 & 271.0 & 24.0 & 2.02 \\
Wogonoside & 461.2 & 285.0 & 24.0 & 2.29 \\
Wogonin & 285.0 & 270.0 & 33.0 & 2.95 \\
Loganin & 408.2 & 229.0 & 12.0 & 1.38 \\
Puerarin & 417.2 & 296.9 & 32.0 & 1.32
\end{tabular}


performed using Analyst 1.5.2 software and MultiQuant2.1.1 software (Applied Biosystems, Foster City, CA, USA).

\section{Drug administration and sampling}

Twelve male rats were divided randomly into two groups with six rats in each. The diabetic model group rats were induced by the streptozotocin (STZ) method as described previously. ${ }^{28}$ Briefly, the diabetic rats were intraperitoneally injected with STZ freshly dissolved in a citrate buffer $\left(0.1 \mathrm{~mol} \mathrm{~L}^{-1}, \mathrm{pH} 4.5\right)$ at a single dosage of $45 \mathrm{mg} \mathrm{kg}^{-1}$ rat weight. The tail blood glucose value was measured 1 week later. Rats presenting blood-glucose levels greater than $16.7 \mathrm{mmol} \mathrm{L}^{-1}$ were put into the diabetic group. All experiments were performed on the tenth day after the injection of STZ or vehicle. The animals were fasted for $12 \mathrm{~h}$ with free access to water before the oral administration of Gandi capsule.

\section{Sample processing}

Internal standard was dissolved with methanol. For plasma samples, an aliquot of $200 \mu \mathrm{L}$ of the IS working solution ( $2 \mathrm{ng}$ $\mathrm{mL}^{-1}$ in methanol) was added to $50 \mu \mathrm{L}$ of the plasma sample. The tubes were vortex mixed for $1.0 \mathrm{~min}$. After centrifugation at 14000 for $10 \mathrm{~min}$, an aliquot of $2 \mu \mathrm{L}$ supernatant was injected into the LC-MS/MS system.

\section{Method validation}

The method validation assays for quantification of baicalin, wogonoside, wogonin and loganin in plasma were performed according to the Guidance for Industry, Bioanalytical Method Validation (https:/www.fda.gov/downloads/Drugs/GuidanceComplianceRegulatoryInformation/Guidances/UCM070107.pdf). The bioanalytical method was validated for selectivity, linearity, low limit of determination (LLOQ), precision, accuracy, recovery, matrix effect, dilution effect and stability. Quality control (QC) samples were prepared at low, medium and high concentrations: 5,50 , and $400 \mathrm{ng} \mathrm{mL}^{-1}$ for baicalin and loga-

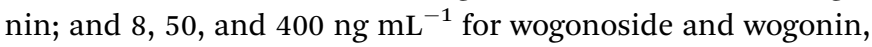
respectively. The linearity of the assay for rat plasma was assessed by analyzing the calibration curves using least-squares linear regression of the peak area ratios of the analytes to the IS versus the nominal concentration of the calibration standard with a weighed factor $\left(1 / C^{2}\right)$. A standard curve calibration was performed at concentrations of 1, 2, 5, 10, 20, 50, 100, and 500 $\mathrm{ng} \mathrm{mL}^{-1}$ for baicalin; $5,7.5,10,20,50,100,200$, and $500 \mathrm{ng}$ $\mathrm{mL}^{-1}$ for wogonoside and wogonin; and 2, 5, 10, 20, 50, 100, 200 , and $500 \mathrm{ng} \mathrm{mL} \mathrm{m}^{-1}$ for loganin each time before the samples were injected into the LC-MS/MS system.

\section{Pharmacokinetic analysis}

All animal procedures were performed in accordance with the Guidelines for Care and Use of Laboratory Animals of Shanghai Jiao Tong University School of Medicine and approved by the Animal Ethics Committee of Shanghai Jiao Tong University School of Medicine. The capsule was prepared by dissolving the contents (with other excipients) in $0.3 \%$ carboxymethylcellulose sodium solution, which was same as the previous pharmacological studies. ${ }^{29}$ Diabetic and normal rats received Gandi capsule by oral administration at a dose of $80 \mathrm{mg} \mathrm{kg}^{-1}$ (containing $1.08 \%$ baicalin, $0.13 \%$ wogonoside, $0.04 \%$ wogonin, and $0.10 \%$ loganin). Six rats were used in each group. Blood samples $(150 \mu \mathrm{L})$ were collected into heparinized Eppendorf tubes at predose $(0 \mathrm{~h})$ and at $0.17,0.5,1.0,1.5,2.0,2.5,3.0,4.0$, $6.0,12.0,24.0$, and $48.0 \mathrm{~h}$ after administration, the whole blood samples were centrifuged at $3000 \mathrm{rpm}$ for $10 \mathrm{~min}$, and the plasma samples were immediately centrifuged and stored at $-20{ }^{\circ} \mathrm{C}$ until analysis.

\section{Statistical analysis}

Pharmacokinetic parameters were calculated using Drug and Statistic (DAS) 2.0 pharmacokinetic software (Chinese Pharmacological Society, Shanghai, China). The data of area under the curve (AUC), maximum concentration $\left(C_{\max }\right)$, terminal halflife $\left(T_{1 / 2}\right)$, time to maximum concentration $\left(T_{\max }\right)$, and mean residence time (MRT) are presented as the mean \pm standard deviation (SD). Comparisons of the pharmacokinetic data were performed by variance (Student' $t$-test) and the statistically significant difference was set at values of $P<0.05$ and $P<0.01$ (SPSS statistical software package, Version 20.0, SPSS Inc., Chicago, IL, USA).

\section{Results and discussions}

\section{Specificity}

The retention times were as follows: baicalin (2.02 $\mathrm{min})$, wogonoside (2.29 $\mathrm{min})$, wogonin (2.95 $\mathrm{min})$, loganin (1.38 $\mathrm{min})$, and puerarin $(1.32 \mathrm{~min}) .[\mathrm{M}+\mathrm{H}]^{+}$ion was chosen for baicalin, wogonoside, wogonin and puerarin. For loganin, $\left[\mathrm{M}+\mathrm{NH}_{4}\right]^{+}$ion was chosen for the quantification. In pre-experiment, loganin with $m / z 408.2\left(\left[\mathrm{M}+\mathrm{NH}_{4}\right]^{+}\right)$was found to be a better spectral response than $m / z 391.16\left([\mathrm{M}+\mathrm{H}]^{+}\right)$. The LLOQ in $\left[\mathrm{M}+\mathrm{NH}_{4}\right]^{+}$ ion was lower than $[\mathrm{M}+\mathrm{H}]^{+}$ion. Representative plasma chromatographs are shown in Fig. 2, including blank plasma, methanol spiked with IS and standards, plasma spiked with mixed standards (LLOQ) and plasma samples obtained $2 \mathrm{~h}$ after oral administration of Gandi capsule. The total retention time was less than 4.3 minutes. LLOQs in positive-ionization mode were set here appropriate for quantitative detection of analytes in the pharmacokinetic studies.

The active ingredients of Gandi capsules which composed of several herbs are very complex. In pre-experiment, about sixteen active compounds, which contained baicalin, wogonoside, wogonin, loganin, rutin, rehmannioside $\mathrm{D}$, astragaloside IV, quercetin, isoquercetin, isoastragaloside, scutellarin, scutellarein, sweroside, ferulic acid, caffeic acid and isoacteoside, have been detected by LC/MS/MS. Simply, the assay was performed with a validated LC/MS/MS system as same as shown in Apparatus and LC-MS/MS conditions part. The main parameters as selected multiple reaction monitoring parameters, collision energies (CE) and LLOQs of the sixteen ingredients were shown in Table S1 (ESI $\dagger$ ). The LLOQsof the all analytes were less than 5 $\mathrm{ng} \mathrm{mL}^{-1}$ in rat plasma. Unfortunately, after oral administration 


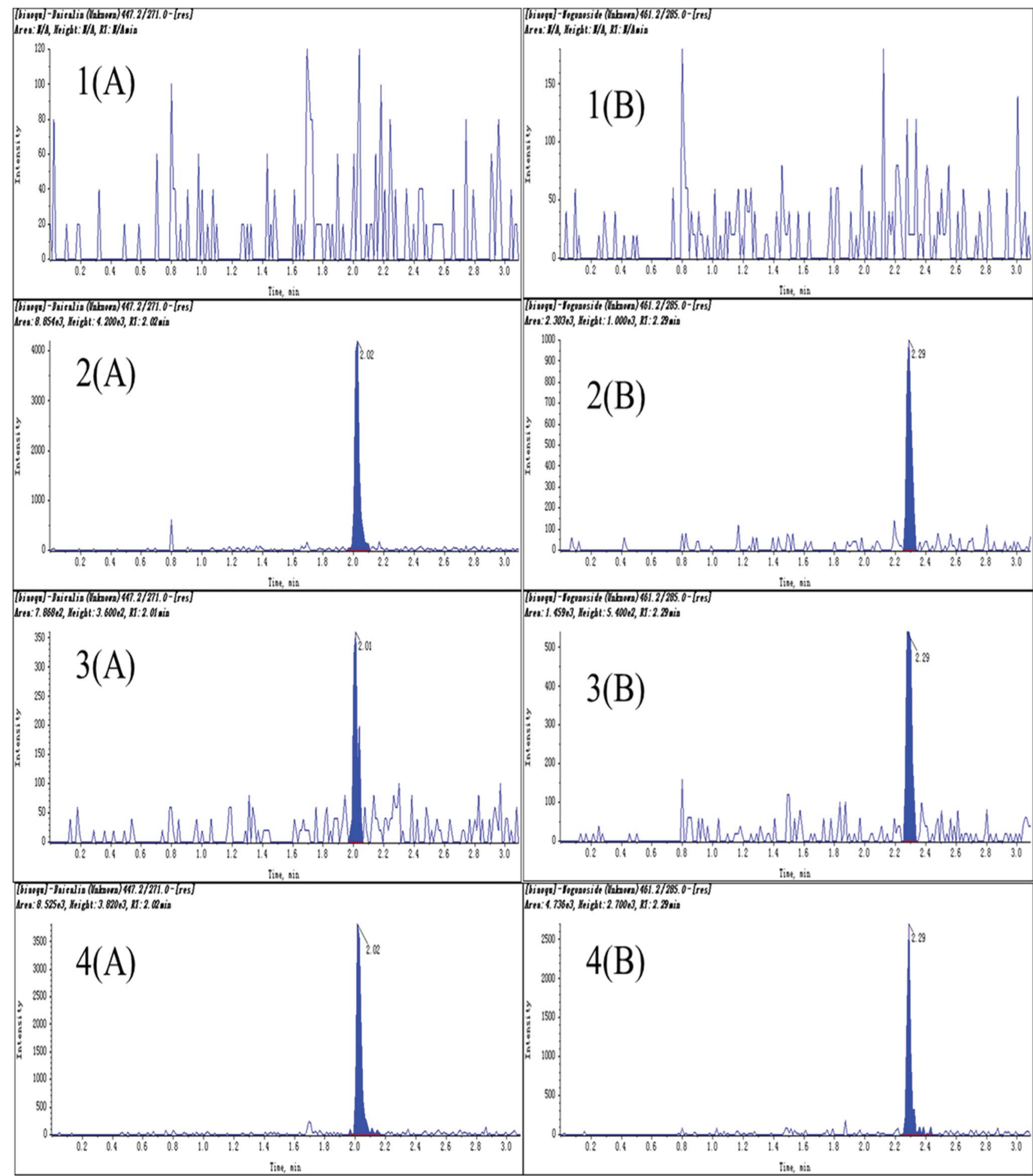

Fig. 2 Representative chromatograms of (A) baicalin, (B) wogonoside, (C) wogonin, (D) loganin and (E) IS in (1) blank plasma, (2) methanol spiked with the analytes $A\left(5.0 \mathrm{ng} \mathrm{mL}^{-1}\right), B\left(2.0 \mathrm{ng} \mathrm{mL}^{-1}\right), C\left(2.0 \mathrm{ng} \mathrm{mL}^{-1}\right), D\left(10.0 \mathrm{ng} \mathrm{mL}^{-1}\right)$ and IS, respectively, (3) blank plasma spiked with the analytes $\mathrm{A}, \mathrm{B}, \mathrm{C}, \mathrm{D}$ at levels of LLOQ and IS, respectively, and (4) a plasma sample from a model rat $2 \mathrm{~h}$ after oral administration of Gandi capsule (722.88 $\mathrm{ng}$ $\mathrm{mL}^{-1}$ for $\mathrm{A}, 221.98 \mathrm{ng} \mathrm{mL}^{-1}$ for $\mathrm{B}, 54.90 \mathrm{ng} \mathrm{mL}^{-1}$ for $C$ and $42.63 \mathrm{ng} \mathrm{mL}^{-1}$ for D).

of Gandi capsule, twelve ingredients could not be found in rat plasma. This result may be explained by low content of the compounds in the preparation or the poor bio availability in rats after oral administration. In fact, the content of most compounds like rehmannioside D, astragaloside IV, isoquercetin, isoastragaloside, scutellarin, scutellarein, sweroside and isoacteoside is too low that less than $0.003 \%$ of the whole prescription. In addition, some active ingredients have been 


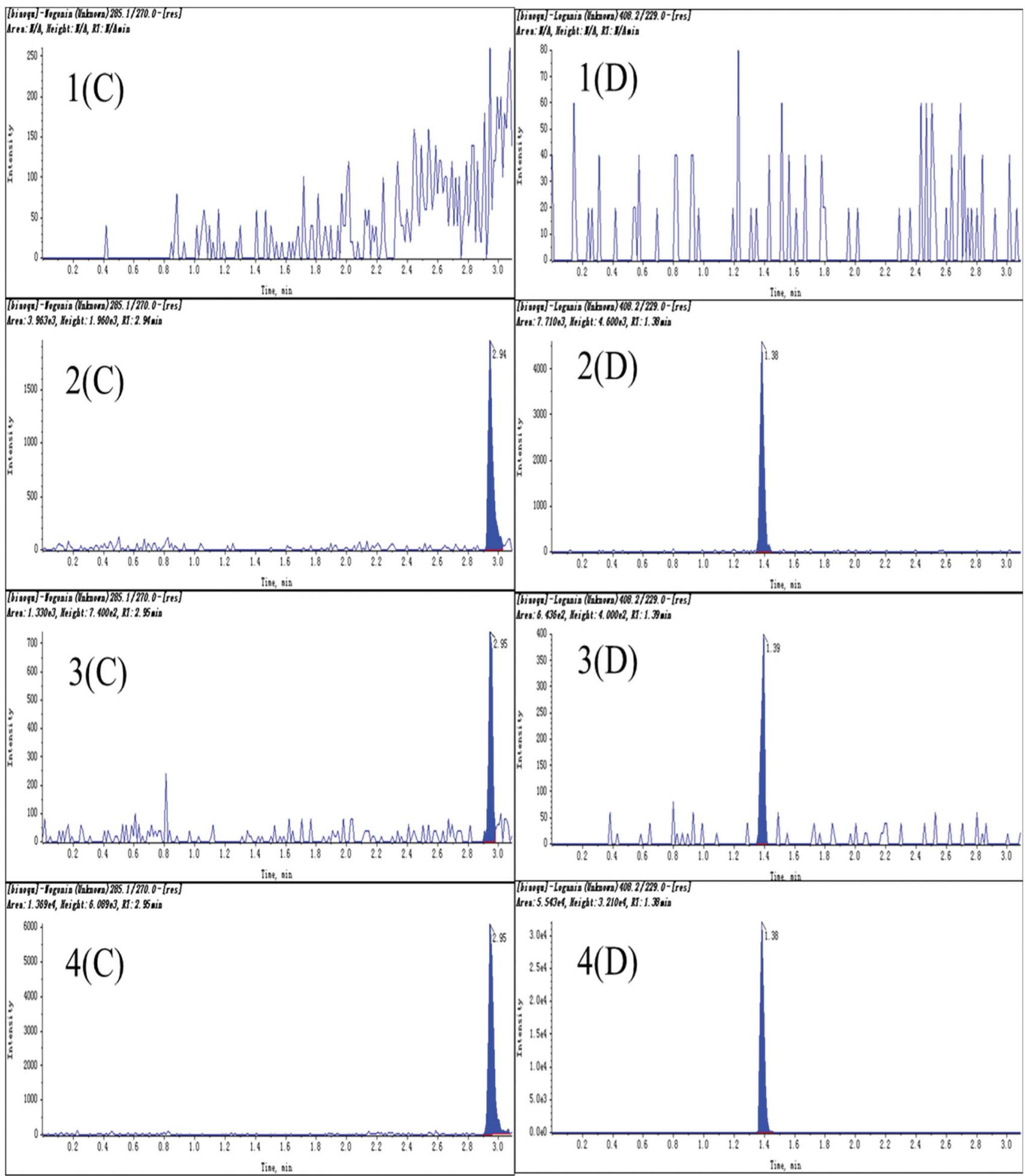

Fig. 2 (contd).

reported with strong metabolism in gastrointestinal tract and liver of rats like quercetin, rutin, ${ }^{30,31}$ caffeic acid and ferulicacid..$^{32,33}$ The first pass effect led to the poor bio availability of the active compounds. These reasons mentioned above turned to the result that the plasma concentration in $48 \mathrm{~h}$ of most ingredients was lower than LLOQs by LC/MS/MS, and pharmacokinetic parameters could not be obtained.

\section{Calibration curves and LLOQ}

The calibration curves, linear ranges, correlation coefficients and LLOQs of baicalin, wogonoside, wogonin and loganin in 


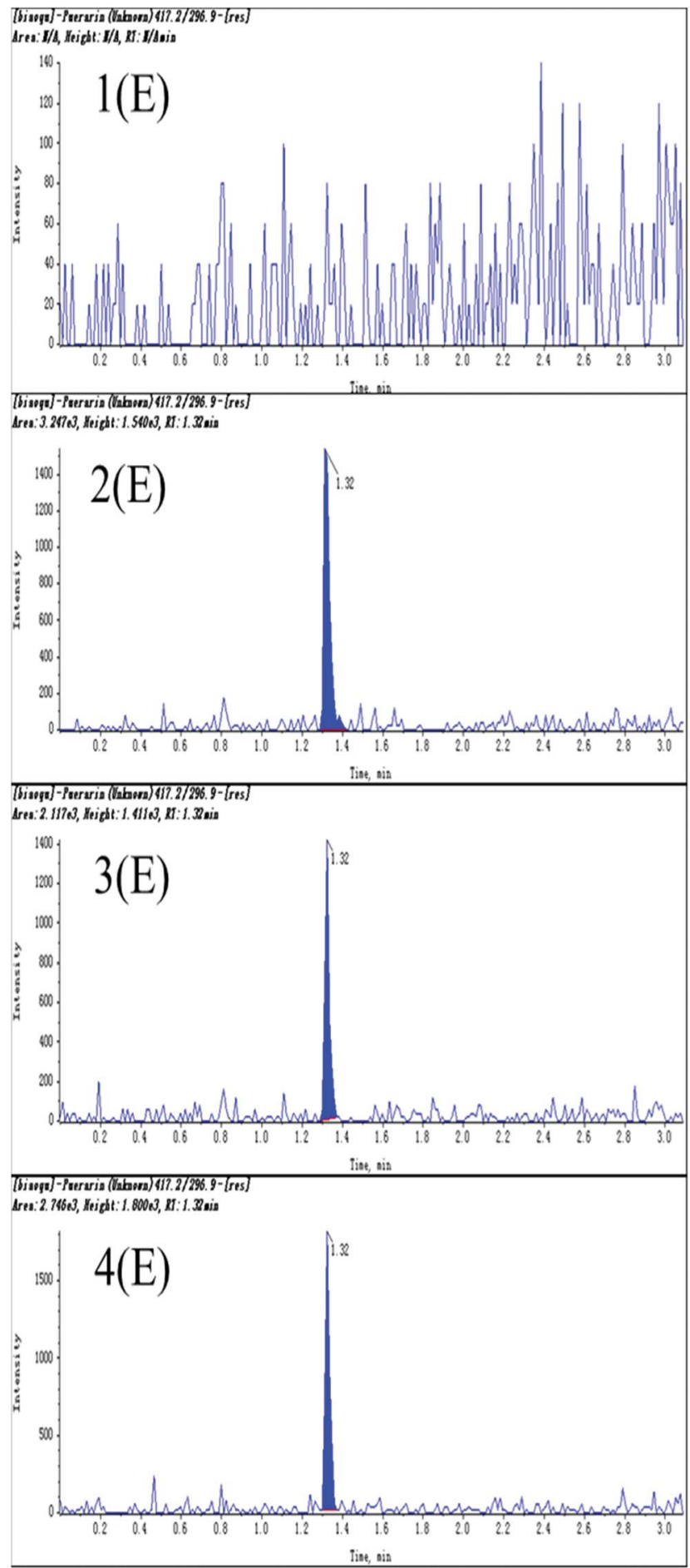

Fig. 2 (contd).

plasma are listed in Table 2. The calibration curves showed good linearity in the corresponding range for the four compounds $\left(r^{2}>0.99\right)$.

\section{Precision, accuracy, extraction recovery and matrix effect}

The precision, accuracy, extraction recovery and matrix effect were obtained by analyzing six QC samples at low, medium, and high concentrations, respectively. The results are given in
Table 3. Precision was measured based on the intra- and interday variability. Intra- and inter-day precisions were determined by analyzing six standard solutions at three concentrations within a single day and on three different days, respectively. The measured concentrations of QC samples were calculated from the calibration curves. The intra- and inter-day precisions and accuracy fit the regulation exceeded $\pm 10 \%$. The extraction recovery was calculated by dividing the peak area of standards 
Table 2 Standard curves, correlation coefficients, LLOQ and linear ranges of lactones in plasma samples

\begin{tabular}{|c|c|c|c|c|}
\hline Biological samples & Calibration curves & Correlation coefficients $\left(r^{2}\right)$ & LLOQ (ng mL $\left.{ }^{-1}\right)$ & Linear range $\left(\mathrm{ng} \mathrm{mL} \mathrm{L}^{-1}\right)$ \\
\hline Baicalin & $y=0.2107 x+0.0583$ & 0.9982 & 1.0 & $1-500$ \\
\hline Wogonoside & $y=0.0777 x-0.0888$ & 0.9986 & 5.0 & $5-500$ \\
\hline Loganin & $y=0.0421 x+0.1818$ & 0.9991 & 2.0 & $2-500$ \\
\hline
\end{tabular}

Table 3 Precision, accuracy extraction recovery and matrix effect of QC samples in rat plasma $(n=6)$

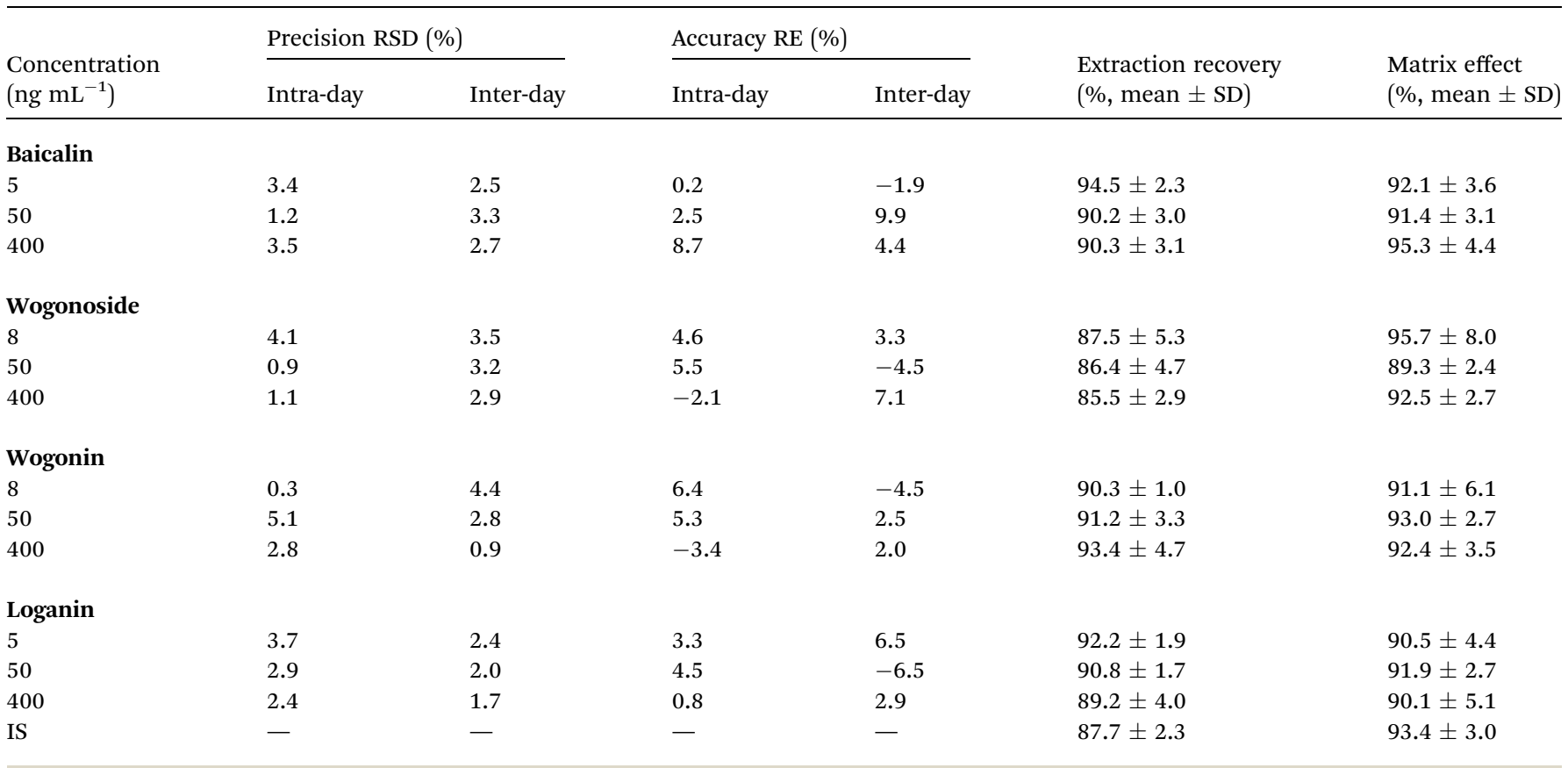

spiked prior to the extraction by that of the four compounds and IS spiked post-extracted sample. Matrix effect was calculated by dividing the peak area of four compounds and IS in the methanol-dissolved samples by that of samples spiked postextraction. The mean extraction recoveries and matrix effect of four analytes were determined by using six replicates of QC samples of each level. The results are displayed in Table 3 . The mean extraction recoveries of the four analytes were greater than $85.5 \%$. The matrix effect of the six analytes ranged from $90.1 \%$ to $95.7 \%$ at the three QC levels, and the extraction recovery and matrix effect of IS were $87.7 \%$ and $93.4 \%$, which indicated that there was no significant matrix effect affecting the quantification of the analytes and IS.

\section{Dilution effect and sample stability}

Dilution effect was investigated to ensure that dilution with blank matrix would not affect the final concentration. Baicalin and wogonoside spiked rat plasma samples were prepared at $5.0 \mu \mathrm{g} \mathrm{mL} \mathrm{m}^{-1}$ and then diluted with pooled rat biological samples at dilution factors of 10 and 50 in six replicates before analysis. The results from six replicates showed satisfying accuracy and reproducibility, and therefore indicated that such a method using methanol precipitation could be applied to the entire scope of concentrations tested in this study (data not shown).

For the measurement of sample stability, three QC samples of low, medium and high concentrations were prepared for analysis under different storage conditions, including shortterm stability at room temperature for $4 \mathrm{~h}$, long-term stability
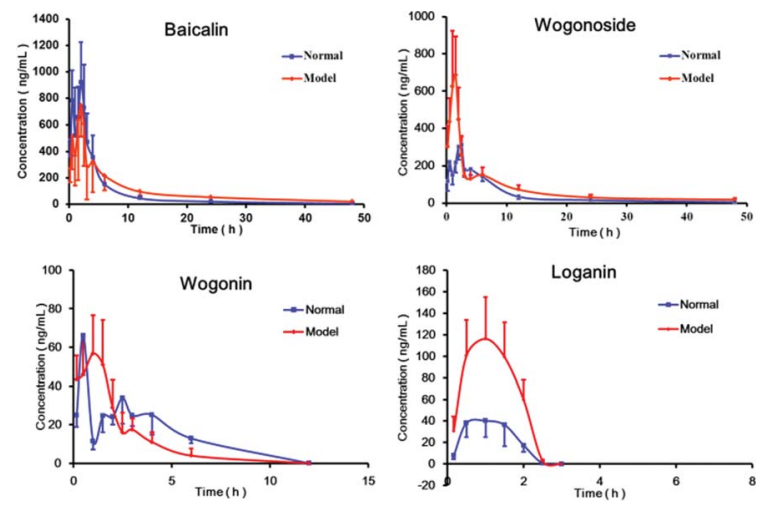

Fig. 3 Mean plasma concentration-time curves of four components after oral administration of Gandi capsule to normal rats $(n=6)$ and diabetic rats $(n=6)$. 
Table 4 Pharmacokinetic parameters of the four compounds in rats $(n=6)^{a}$

\begin{tabular}{lllcccc}
\hline Compounds & Group & $T_{\max }(\mathrm{h})$ & $C_{\max }\left(\mathrm{ng} \mathrm{mL}{ }^{-1}\right)$ & $T_{1 / 2} z(\mathrm{~h})$ & $\mathrm{MRT}_{0-48}(\mathrm{~h})$ & $\mathrm{AUC}_{0-48}(\mathrm{ng} \mathrm{L} \mathrm{h})$ \\
\hline \multirow{2}{*}{ Baicalin } & Model & $2.00 \pm 0.71$ & $1241.55 \pm 341.04$ & $9.50 \pm 3.19^{*}$ & $9.98 \pm 0.28^{* *}$ & $5104.52 \pm 860.78^{* *}$ \\
& Normal & $1.50 \pm 1.40$ & $1250.55 \pm 123.81$ & $9.45 \pm 2.87$ & $4.72 \pm 0.39$ & $3801.16 \pm 599.75$ \\
Wogonoside & Model & $1.25 \pm 0.35$ & $1397.70 \pm 461.74^{*}$ & $11.90 \pm 1.24^{*}$ & $10.09 \pm 1.76^{*}$ & $4010.04 \pm 867.08^{*}$ \\
& Normal & $2.00 \pm 0.71$ & $406.35 \pm 166.95$ & $11.32 \pm 2.79$ & $9.49 \pm 0.16$ & $2550.66 \pm 799.78$ \\
Wogonin & Model & $1.25 \pm 0.35$ & $101.05 \pm 26.52$ & $2.87 \pm 1.23$ & $2.94 \pm 1.73$ & $241.36 \pm 110.93^{*}$ \\
& Normal & $0.50 \pm 0.00$ & $66.40 \pm 17.25$ & $1.52 \pm 0.25$ & $1.22 \pm 0.54$ & $142.64 \pm 13.80$ \\
Loganin & Model & $1.00 \pm 0.00$ & $118.00 \pm 53.74^{* *}$ & $0.65 \pm 0.42$ & $1.16 \pm 0.02^{*}$ & $188.83 \pm 80.87^{* *}$ \\
& Normal & $1.25 \pm 0.35$ & $42.05 \pm 15.55$ & $0.46 \pm 0.38$ & $1.12 \pm 0.03$ & $64.28 \pm 28.40$
\end{tabular}

${ }^{a}$ Data are presented as the mean $\pm \mathrm{SD}$. Statistical significance indicated by ${ }^{*} P<0.05$ versus the normal group. ${ }^{* *} P<0.01$ versus the normal group.

at $-80{ }^{\circ} \mathrm{C}$ for 30 days, three freeze-thaw cycles at $-80{ }^{\circ} \mathrm{C}$ and in autosampler for $24 \mathrm{~h}$ at $4{ }^{\circ} \mathrm{C}$. The results suggested that the established method was suitable for the pharmacokinetic study.

\section{Plasma pharmacokinetic study}

The validated LC/MS/MS method was applied to a pharmacokinetic study after oral administration of Gandi capsule to normal and diabetic rats. The mean plasma concentration versus time profiles of four compounds in normal and diabetic rats are illustrated in Fig. 3. The main pharmacokinetic parameters are summarized in Table 4. Because of the quick elimination phase, loganin can only be detected in 2.5 hours after oral administration.

As shown in Fig. 3 and Table 4, the $C_{\max }$ values of wogonoside and loganin in the diabetic rats were $344.1 \%$ and $277.65 \%$ in relative to those in the normal rats, respectively. Compared with normal rats, the $\mathrm{AUC}_{0-48} \mathrm{~h}$ of the four compounds in the diabetic rats increased sharply. The $T_{1 / 2}$ value of baicalin and wogonoside for the diabetic rats was longer of that of the normal rats. Compared to those of the normal group, MRT values of baicalin, wogonoside and loganin increased remarkably in the diabetic rats. The results pointed out that the absorption of the four active components was increased, whereas the distribution and elimination processes were slowed down in the diabetic rats compared with the normal rats.

Accumulating evidence indicates that the pharmacokinetic behaviors of drugs may change under the condition of diabetes mellitus. However, the underlying mechanism remains unclear. Diabetic mellitus can induce several complications such as nephropathy and liver disease, ${ }^{34}$ and may further affect the metabolism and elimination of some drugs in vivo, and this may lead to the pharmacokinetic parameters such as AUC, $T_{1 / 2}$ and MRT being higher in diabetic rats than in normal rats. ${ }^{27}$

Some papers had reported that the pharmacokinetic profile of baicalin, wogonoside and loganin involved the drug metabolizing enzymes (such as $\beta$-glucuronidase, UDPglucuronosyltransferase, cytochrome P450) and drugtransporters (such as MRP 2 and P-glycoprotein). ${ }^{35,36}$ However, the function and expression of many drug metabolizing enzymes and drug transporter had been reported to be altered when in the pathological state of diabetes mellitus. ${ }^{37,38}$ These mentioned above might result in the alteration of pharmacokinetic behavior of the three compounds in diabetic rats following oral administration of Gandi capsule when compared with that in normal rats.

Wogonin was found in Gandi capsule, but it also could be the metabolite of wogonoside in vivo. In fact, previous published papers demonstrated that wogonoside was first hydrolyzed to its a glycon wogonin by intestinal bacteria in the intestinal tract and then wogonin was absorbed into intestinal mucosa to transform into wogonoside again. ${ }^{39,40}$ Gut microbiota in diabetic rats may be different from normal group. Thus, the pharmacokinetic parameters result of wogonoside was similar to the phenomenon observed in another previously published study. ${ }^{\mathbf{4 1 , 4 2}}$ As the metabolite of wogonoside, the concentration time profile for wogonin could change with wogonoside $\left(\mathrm{AUC}_{0-}\right.$ $48 \mathrm{~h})(P<0.05)$. These rational explanations still may not be accurate due to lack of direct evidence about how intestinal bacteria affect the metabolism of wogonin.

In conclusion, the pharmacokinetic behavior of baicalin, wogonoside, wogonin and loganin (especially the systemic exposure [AUC]) was significantly altered in diabetic rats after orally administrated of Gandi capsule, which may partly result from the changed activity of drug metabolizing enzymes and drug transporters under the pathological state of diabetes mellitus. The results presented a meaningful basis for the clinical applications of Gandi capsule when used in the treatment of diabetes mellitus.

\section{Conclusions}

In the present study, an LC-MS/MS method for the simultaneous quantification of four active ingredients in rat plasma has been developed and validated. The method was successfully applied to a comparison of the pharmacokinetic behaviors of the four compounds in rat plasma between normal and diabetic rats after oral administration of Gandi capsule. The results indicated that the main active ingredient had a better absorption in diabetic rats than in the normal rats, which could help to explain the in vivo processes and mechanism of action of Gandi capsule. The use of Gandi capsule could play an important role in diabetes mellitus treatment in the future.

\section{Conflicts of interest}

There are no conflicts to declare. 


\section{Acknowledgements}

This work was supported by Natural Science Foundation of Shanghai (15401901600, 16401900500).

\section{References}

1 X. Wei, H. J. Yao, Y. Liu and Z. Jian, China Pharm., 2016, 27, 225-227.

2 W. R. Zhu, Y. H. Yang, L. Zheng and X. H. Shen, Chinese Journal of Current Traditional and Western Medicine, 2005, 3, 1153.

3 J. Zhang, Y. N. Tang, T. Chen, Y. Liu and Y. F. Chai, China Pharm., 2014, 25, 2921-2923.

4 C. Ye, S. L. Li, W. X. Yao, Y. S. Qiu, Y. Liu, Z. Y. Wu and Y. Q. Hou, Innate Immun., 2016, 22, 196-204.

5 Y. Z. Yang, Y. Z. Tang and Y. H. Liu, J. Ethnopharmacol., 2013, 148, 271-276.

6 S. Ikemoto, K. Sugimura, N. Yoshida, R. Yasumoto, S. Wada, K. Yamamoto and T. Kishimoto, Urology, 2000, 55, 951-955.

7 S. J. Kim, H. J. Kim, H. R. Kim, S. H. Lee, S. D. Cho, C. S. Choi, J. S. Nam and J. Y. Jung, Mol. Med. Rep., 2012, 6, 1443-1449. 8 L. Yang, Q. Wang, D. X. Li, Y. Q. Zhou, X. L. Zheng, H. Sun, J. Q. Yan, L. Zhang, Y. Lin and X. Wang, Apoptosis, 2013, 18, 618-626.

9 D. L. Huynh, N. Sharma, A. K. Singh, S. S. Sodhi, J. J. Zhang, R. K. Mongre, M. Ghosh, N. Kim, Y. H. Park and D. K. Jeong, Chin. J. Nat. Med., 2017, 15, 15-40.

10 H. Vaez, M. Arab, A. Delazar and M. A. Eghbal, Trends Pharmacol. Sci., 2015, 1, 139-148.

11 B. R. Zhou, H. B. Yin, Y. Xu, D. Wu, Z. H. Zhang, Z. Q. Yin, F. Permatasari and D. Luo, Free Radical Res., 2012, 46, 1458-1471.

12 D. Shieh, L. T. Liu and C. C. Lin, Anticancer Res., 2000, 20, 2861-2865.

13 P. Ma, X. Y. Mao, X. L. Li, Y. D. Qiao, Z. Q. Liu, H. H. Zhou and Y. G. Cao, Mol. Med. Rep., 2015, 12, 6377-6383.

14 S. Chirumbolo and G. Bjørklund, Environ. Toxicol. Pharmacol., 2017, 51, 156-157.

15 C. M. Lee, H. A. Jung, S. H. Oh, C. H. Park, T. Tanaka, T. Yokozawa and J. S. Choi, Arch. Pharmacal Res., 2015, 38, 1090-1098.

16 C. H. Park, T. Tanaka, J. H. Kim, E. J. Cho, J. C. Park, N. Shibahara and T. Yokozawa, Toxicology, 2011, 290, 14-21.

17 K. Liu, H. Xu, G. Lv, B. Liu, M. K. Lee, C. Lu, X. Lv and Y. Wu, Life Sci., 2015, 123, 78-85.

18 J. W. Wang, M. H. Chiang, C. M. Lu and T. H. Tsai, J. Chromatogr. B: Anal. Technol. Biomed. Life Sci., 2016, 1026, 152-161.

19 Y. Du, B. He, Q. Li, J. He, D. Wang and K. Bi, J. Sep. Sci., 2017, 40, 2097-2106.

20 M. J. Kang, G. S. Ko, D. G. Oh, J. S. Kim, K. Noh, W. Kang, W. K. Yoon, H. C. Kim, H. G. Jeong and T. C. Jeong, Arch. Pharmacal Res., 2014, 37, 371-378.
21 Y. Cai, S. Li, T. Li, R. Zhou, A. T. Wai and R. Yan, J. Chromatogr. B: Anal. Technol. Biomed. Life Sci., 2016, 1026, 124-133.

22 J. M. M. Salmani, X. Wu, J. A. Jacob, R. Fu and B. Chen, Acta Pharm., 2017, 67, 373-384.

23 M. Zhao, J. Tao, D. Qian, P. Liu, E. X. Shang, S. Jiang, J. Guo, S. L. Su, J. A. Duan and L. Du, J. Chromatogr. B: Anal. Technol. Biomed. Life Sci., 2016, 1009, 122-129.

24 S. Kataoka, H. Yasui, M. Hiromura and H. Sakurai, Life Sci., 2005, 77, 2814-2829.

25 Y. X. Deng, Q. Z. Shi, B. Chen, X. J. Zhang, S. Z. Liu and X. M. Qiu, Fitoterapia, 2012, 83, 1435-1442.

26 X. Y. Zeng, S. Dong, N. N. He, C. J. Jiang, Y. Dai and Y. F. Xia, Fitoterapia, 2015, 105, 119-126.

27 B. B. Wei, Z. X. Chen, M. Y. Liu and M. J. Wei, Molecules, 2017, 22, 1267.

28 Q. Q. Wang, J. Zhang, Z. F. Pi, Z. Zheng, J. P. Xing, F. R. Song, S. Liu and Z. Q. Liu, Anal. Methods, 2015, 7, 45-52.

29 Y. Xie, Z. H. Jiang, H. Zhou, W. Z. Ma, Y. F. Wong, Z. Q. Liu and L. Liu, Biomed. Chromatogr., 2014, 28, 1294-1302.

30 A. K. Kammalla, M. K. Ramasamy, J. Chintala, G. P. Dubey, A. Agrawal and I. Kaliappan, Eur. J. Drug Metab. Pharmacokinet., 2015, 40, 277-284.

31 S. H. Bang, Y. J. Hyun, J. Shim, S. W. Hong and D. H. Kim, J. Microbiol. Biotechnol., 2015, 25, 18-25.

32 J. Li, Y. Bai, Y. Bai, R. Zhu, W. Liu, J. Cao, M. An, Z. Tan and Y. X. Chang, Evid. Based Compl. Alt., 2017, 2017.

33 M. H. Omar, W. Mullen, A. Stalmach, C. Auger, J. M. Rouanet, P. L. Teissedre, S. T. Caldwell, R. C. Hartley and A. Crozier, J. Agric. Food Chem., 2012, 60, 5205-5214.

34 A. Kumar, S. K. Bharti and A. Kumar, Apollo Medicine, 2014, 11, 161-166.

35 M. Zhao, J. Tao, D. Qian, P. Liu, E. X. Shang, S. Jiang, J. Guo, S. L. Su, J. A. Duan and L. Du, J. Chromatogr. B: Anal. Technol. Biomed. Life Sci., 2016, 1009, 122-129.

36 M. Y. He, Y. X. Deng, Q. Z. Shi, X. J. Zhang and Y. Lv, J. Ethnopharmacol., 2014, 155, 334-342.

37 S. J. Oh, J. M. Choi, K. U. Yun, J. M. Oh, H. C. Kwak, J. G. Oh, K. S. Lee, B. H. Kim, T. H. Heo and S. K. Kim, Chem.-Biol. Interact., 2012, 195, 173-179.

38 Y. X. Deng, Q. Z. Shi, B. Chen, X. J. Zhang, S. Z. Liu and X. M. Qiu, Fitoterapia, 2012, 83, 1435-1442.

39 S. H. Xing, M. Y. Wang, Y. Peng, D. F. Chen and X. b. Li, J. Ethnopharmacol., 2014, 152, 183-189.

40 H. S. Kim, J. Y. Kim, M. S. Park, H. Zheng and G. E. Ji, J. Microbiol. Biotechnol., 2009, 19, 1650-1655.

41 M. Y. He, Y. X. Deng, Q. Z. Shi, X. J. Zhang and Y. Lv, J. Ethnopharmacol., 2014, 155, 334-342.

42 A. Sabatino, G. Regolisti, C. Cosola, L. Gesualdo and E. Fiaccadori, Curr. Diabetes Rep., 2017, 17, 16. 